\title{
THE FORMATION OF IN-RICH REGIONS AT THE PERPHERY OF THE INVERTED HEXAHONAL PITS OF InGaN THIN-FILMS GROWN BY METALORGANIC VAPOR PHASE EPITAXY
}

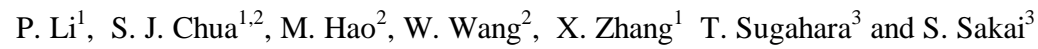 \\ ${ }^{1}$ Center for Opto-electronics \\ Dept. of Electrical Engineering \\ National University of Singapore \\ SINGAPORE 119260 \\ ${ }^{2}$ Institute of Materials Research and Engineering \\ SINGAPORE \\ ${ }^{3}$ Dept. of Electrical and Electronics Engineering, \\ The University of Tokushima, \\ 2-1 Minami-Josanjima 770-8506, \\ Japan
}

\section{ABSTRACT}

InGaN thin films were grown by low-pressure metalorganic-vapor-phase-epitaxy (MOVPE) and characterized by cathodoluminescence (CL) and scanning electron microscopy (SEM). SEM images showed that InGaN samples have inverted hexagonal pits which are formed by the In segregation on the (1011) surfaces. Room temperature $\mathrm{CL}$ at the wavelengths corresponding to the GaN band edge, the In-poor and In-rich regions showed that the In-rich regions formed at the periphery of the hexagonal pits.

\section{INTRODUCTION}

The development of blue LEDs and laser diodes has attracted considerable research activities on the growth of GaN based III-V nitrides. The band gap of InGaN can be varied over nearly the whole spectral range from near UV to red, so it is usually used in active regions of these devices.

The growth of $\operatorname{In}_{\mathrm{x}} \mathrm{Ga}_{1-\mathrm{x}} \mathrm{N}$ alloys is extremely difficult mostly due to the trade-off between the epilayer quality and the amount of $\mathrm{InN}$ incorporation into the alloy. Growth at the temperatures of approximately $800^{\circ} \mathrm{C}$ typically results in high crystalline quality but the amount of $\mathrm{InN}$ in the solid is limited to low values because of the high volatility of In. Lowering the growth temperature results in an increase in the In content at the cost of reduced crystalline quality. The difference in lattice constant and thermal stability between the two constituents, $\mathrm{InN}$ and $\mathrm{GaN}$, also complicates the growth of $\operatorname{In}_{\mathrm{x}} \mathrm{Ga}_{1-\mathrm{x}} \mathrm{N}$. The lattice mismatch can lead to a miscibility gap[1], which causes fluctuations of In content across the film. Singh and co-workers [2] provided strong evidence of phase separation in InGaN thick films grown by MBE. El-Masry et al [3] reported phase separation in thick InGaN films grown by MOVPE. Tran et al [4] showed that when phase-separation occurs, InGaN clusters with different indium compositions can coexist 
and that the high brightness in blue and green LEDs is due to the radiative recombination in the In-rich InGaN clusters.

The mechanism of phase-separation is still not clear. Wu et al [5] reported that the Vdefects initiate at the threading dislocations in one of the quantum wells in a multiple quantum well (MQW) stack and found that V-defect is correlated with the localized excitonic recombination centers that give rise to a long-wavelength shoulder in photoluminescence (PL) and cathodoluminescence (CL) spectra.

Sugahara and Sakai [6] discussed the role of dislocation in InGaN phase separation. They showed that the dislocations in InGaN act as nonradiative recombination centers and confirmed that the phase separation in $\mathrm{InGaN}$ is caused by the spiral growth due to mixed dislocations. They demonstrated that dislocations with the screw component favor the formation of In-rich regions. The extra combinations of the dangling bonds in the dislocated areas can prevent the evaporation of InN during the InGaN growth.

\section{EXPERIMENTAL}

The four samples $(245 \mathrm{~K} 1,245 \mathrm{~K} 1 \mathrm{~A}, 245 \mathrm{~K} 1 \mathrm{~B}, 245 \mathrm{~K} 1 \mathrm{C})$ of $\mathrm{In}_{\mathrm{x}} \mathrm{Ga}_{1-\mathrm{x}} \mathrm{N}$ films were grown by MOVPE (Emcore D125) on (0001) sapphire substrates. MOVPE was conducted using TMGa, TMIn and $\mathrm{NH}_{3}$ as precursors. A $2 \mu \mathrm{m}$ thick undoped bulk $\mathrm{GaN}$ was first grown on the $250 \AA$ thick GaN buffer layer. The growth temperature was $530^{\circ} \mathrm{C}$ and $1050^{\circ} \mathrm{C}$ for the $\mathrm{GaN}$ buffer and bulk layer, respectively. After deposition of the $\mathrm{GaN}$ bulk layer, the growth temperature was lowered down to about $700^{\circ} \mathrm{C}$ for the deposition of $\mathrm{InGaN}$. The InGaN layer thickness was about $500 \AA$. There was no cap layer on the top of InGaN. $\mathrm{H}_{2}$ and $\mathrm{N}_{2}$ were used as carrier gases for the growth of $\mathrm{GaN}$ and $\mathrm{InGaN}$, respectively. The TMIn/TMGa molar ratios were $0.8789,0.89,0.8935$, and 0.8935 , respectively for the four samples. The samples were analyzed by cathodoluminescence (CL), high-resolution $\mathrm{X}$-ray diffraction (HRXRD) and scanning electron microscopy (SEM) at room temperature (RT). CL measurements were performed with an acceleration voltage of 5 or $15 \mathrm{kV}$ using a JOEL 6400 SEM equipped with an Oxford Mono CL2.

\section{RESULTS}

\section{Cathodoluminescence Spectra and Mapping}

Figure 1 shows the room temperature CL spectra of the four samples $(245 \mathrm{k} 1,245 \mathrm{k} 1 \mathrm{~A}$, $245 \mathrm{k} 1 \mathrm{~B}, 245 \mathrm{k} 1 \mathrm{C})$ by scanning the incident electron beam in relatively wide area $\left(10.6 \times 8.1 \mu \mathrm{m}^{2}\right)$. The CL spectra of sample $245 \mathrm{k} 1 \mathrm{~A}$, and $245 \mathrm{k} 1 \mathrm{C}$ exhibit two peaks. The peaks with longer wavelengths were attributed to band-edge related transitions in the Inrich regions. The In-contents of the In-poor regions determined by HRXRD are $16.19 \%$, $16.42 \%, 19.53 \%$ and $20.02 \%$, respectively. In addition to the In-poor regions, In-rich regions with In-contents as high as $51.3 \%$ have been found for the four samples. 


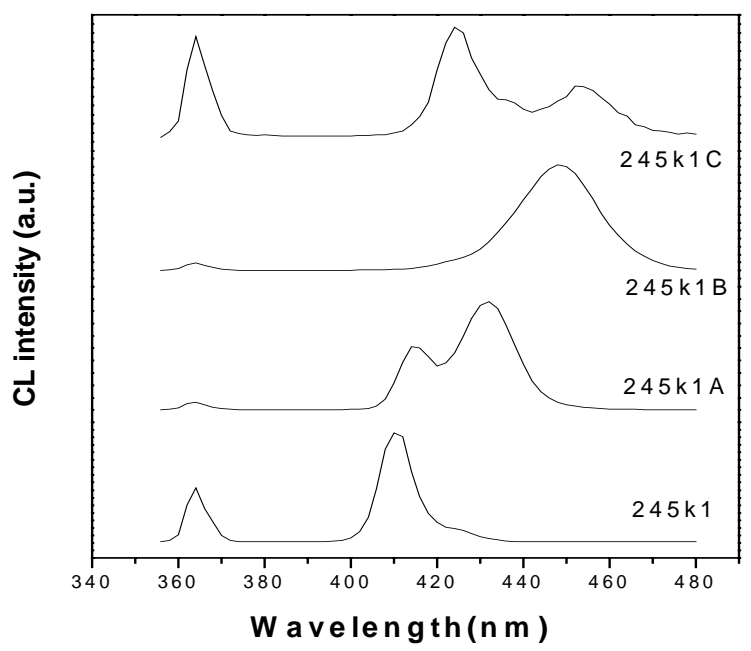

Fig.1. Cathodoluminescence spectra of the four samples, $245 \mathrm{kl}, 245 \mathrm{klA}, 245 \mathrm{klB}$, $245 \mathrm{klC}$ at $R T$.

Figure 2 shows plan-view SEM and CL images taken at wavelengths corresponding to the $\mathrm{GaN}$ band edge $(364 \mathrm{~nm})$, InGaN In-poor regions $(410 \mathrm{~nm}, 414 \mathrm{~nm}, 424 \mathrm{~nm}$, and $424 \mathrm{~nm}$ for sample 245k1, 245k1A, 245k1B, and 245k1C, respectively), and In-rich regions (424nm, $432 \mathrm{~nm}, 448 \mathrm{~nm}$, and $454 \mathrm{~nm}$ for sample 245k1, 245k1A, 245k1B, and 245k1C, respectively).

In the SEM images, all the samples have "inverted hexagonal pits" (IHPs) but with different size and density. Samples grown under higher TMIn/TMGa molar ratios tend to have cathodoluminescence with longer wavelengths and larger IHPs. Sample 245k1C has the largest IHPs with a diameter of approximately $1 \mu \mathrm{m}$.

For any one of the four samples, the $\mathrm{GaN}$ band-edge $\mathrm{CL}$ at $364 \mathrm{~nm}$ is not uniform. The dark spots correspond to non-radiative recombination centers which were reported to be related with dislocations [6]. The CL from the In-poor regions is not uniform either, which demonstrates the non-homogeneous nature of hetero-epitaxially grown InGaN. The CL from In-rich region comes only from the periphery of the IHPs. The situation is most clearly shown in the CL at $454 \mathrm{~nm}$ for the sample $245 \mathrm{k} 1 \mathrm{C}$ where the CL from In-rich regions is associated with the periphery of the two large IHPs.

One may argue that the longer wavelength CL comes from the defects which are captured by dislocations. To further clarify this, we performed temperature-dependent photoluminescence (PL) [7] and excitation-power-dependent PL as well as time-resolved PL (TRPL) [8]. The PL of the four samples have long wavelength peaks or shoulders, the temperature-dependent behavior of the long wavelength peak is S-shaped-like which was reported by Cho et al [9]. The TRPL demonstrated nano-second-order lifetime of the long wavelength peak. This fact excludes the possibility of donor-acceptor-pair transition (DAP) which usually has a longer lifetime in the micro-second order [9]. Based on the above PL experiments and HRXRD, we can confirm that In-rich regions occur in all of our four samples. 

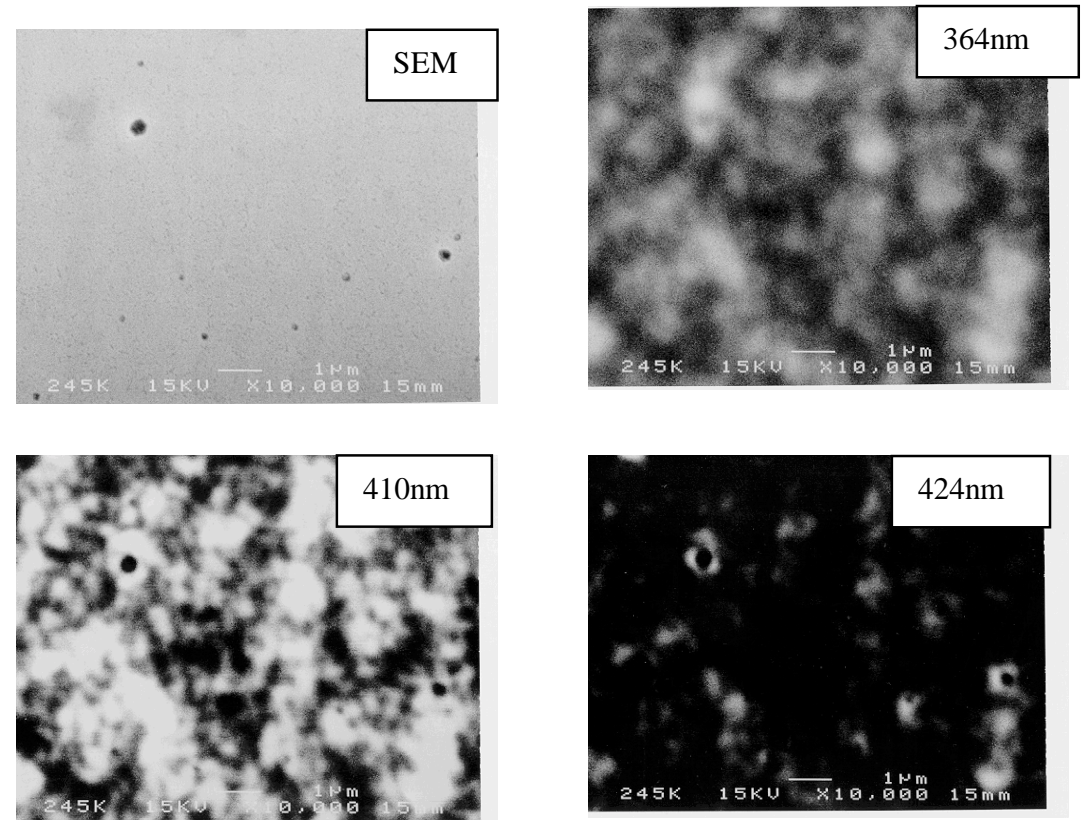

Fig.2-1. Top-view SEM and CL of the sample 245K1
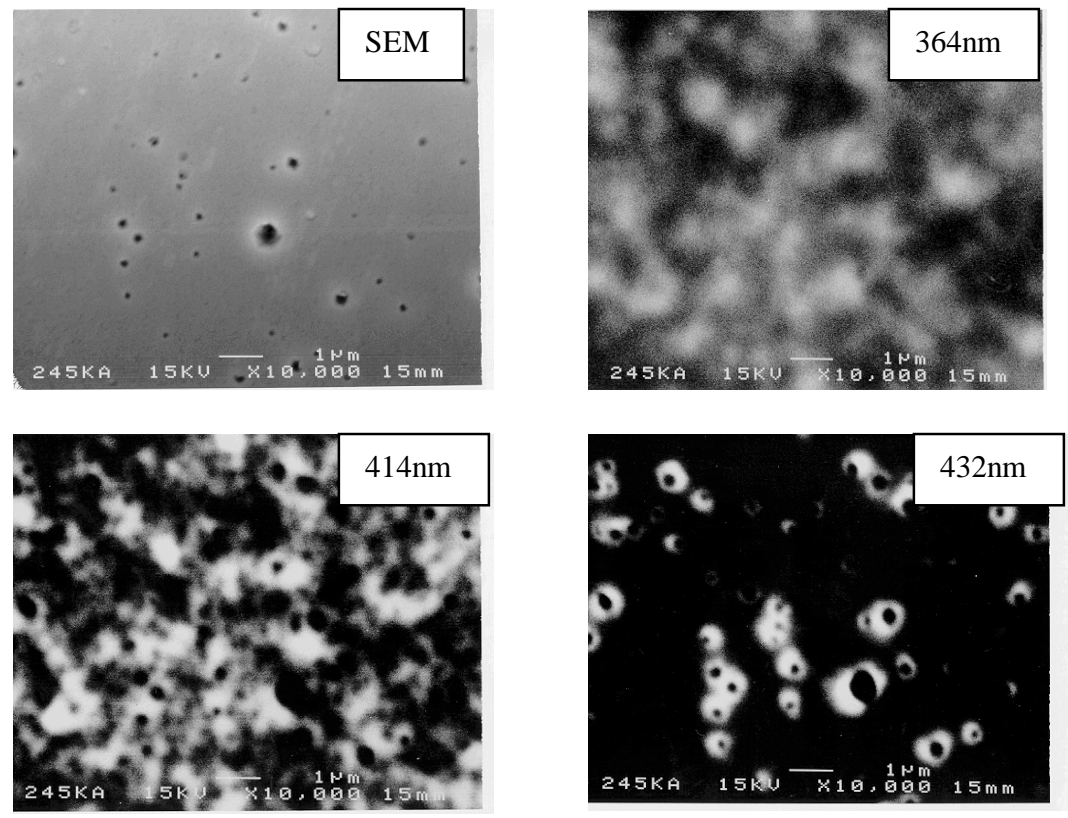

Fig.2-2. Top-view SEM and CL of the sample $245 K 1 \mathrm{~A}$ 

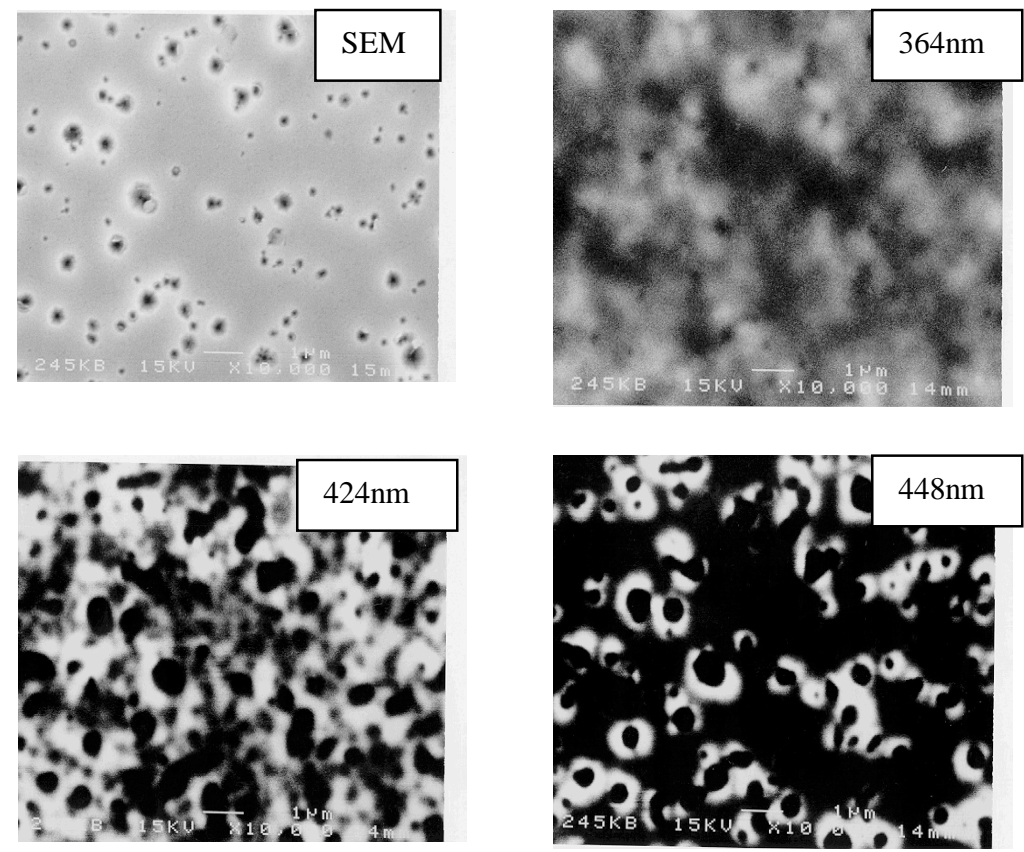

Fig.2-3. Top-view SEM and CL of the sample $245 K 1 B$
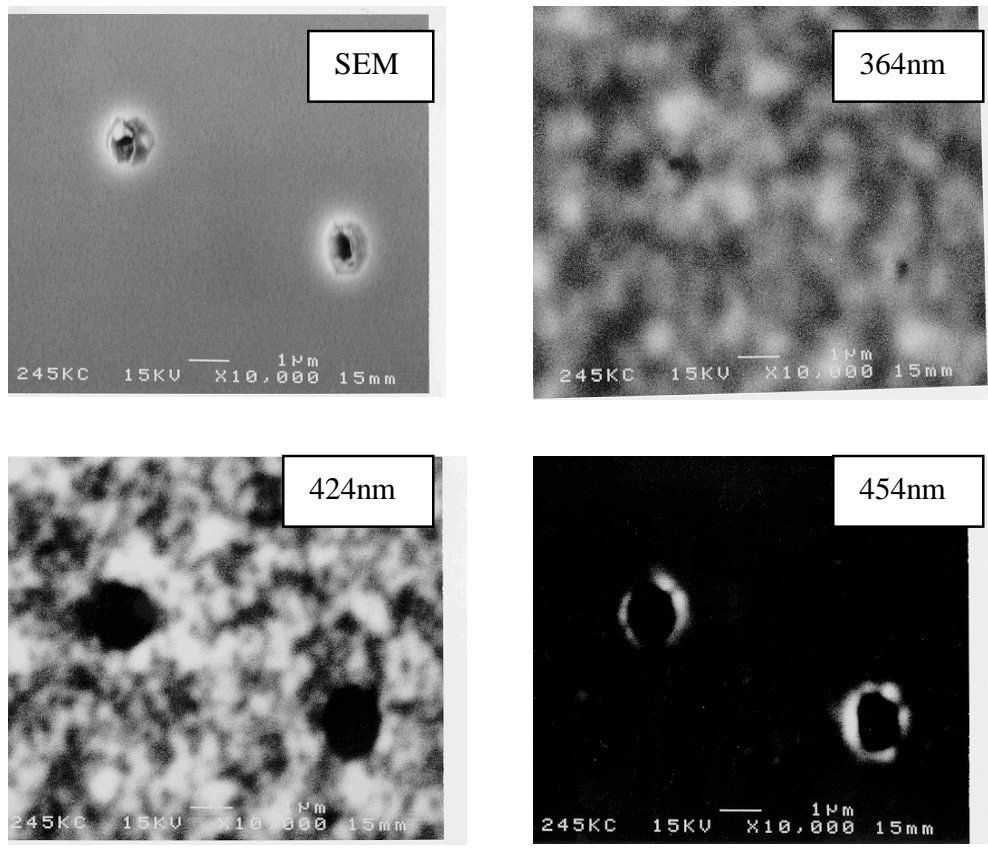

Fig.2-4. Top-view SEM and CL of the sample 245K1C 


\section{DISCUSSIONS}

The formation of IHPs was investigated theoretically by Northrup [10]. A strong preference for In surface segregation and occupation on a specific surface (1011) was demonstrated using first-principle calculations. His calculations indicate that in the absence of In, the equilibrium pit size is on the order of a few nanometers. However, the segregation of In on (1011) surfaces can provide a strong driving force to reduce the surface energy and thereby allow the formation of large IHPs. In fact, In behaves as a differential surfacant, reducing the energy of the (1011) surfact relative to (0001) surface. This effect promotes the formation of larger IHPs. In our samples, these IHPs as large as $1 \mu \mathrm{m}$ in diameter were found. The strong $\mathrm{CL}$ with longer wavelengths at room temperature from the regions near IHPs can be attributed to the quantum-confinement enhanced effect in the form of quantum well or quantum dots with high In content near IHPs. The formation of In-rich region near IHPs can be explained by carefully examining the role of dislocation in the growth of InGaN: the extra dangling bonds in dislocated area can prevent the evaporation of InN during the InGaN growth [7]. Therefore, the dislocations can become the triggering centers for In-rich regions.

It is worthwhile to distinguish the phase-separation (bulk diffusion) process with the segregation that may have occurred during the growth. In this paper, the latter: the In segregation on the growing surface and the preference for InN bonds on (1011) can explain the formation of the In-rich regions at the periphery of IHPs.

\section{Conclusions}

In conclusions, RT CL at wavelengths corresponding to the GaN near band egde, the Inpoor and In-rich regions were performed on the InGaN samples. It was found that the Inrich regions formed at the periphery of the IHPs. The segregation of In on the (1011) surfaces allows the formation of large IHPs.

\section{REFERENCES}

1. I. Ho and G. B. Stringfellow, Appl. Phys. Lett. 69, pp.2701, (1996).

2. R. Singh, D. Doppalapudi, T. D.Moustakas, and L. T. Romano, Appl. Phys. Lett. 70, pp.1089, (1997).

3.N. A. El-Masry, E. L. Piner, S. X. Liu, and S. M. Bedair ,Appl. Phys. Lett., 72, pp.40, (1998).

4. C. Tran, R. Karlicek, M. Schurman, V. Merai, A. Osinsky, Y. Li, I. Eliashevich, M. Brown, J. Nering, T. DiCarlo, I. Ferguson and R. Stall, $2^{\text {nd }}$ Inter. Symp. On Blue Laser and LEDs, Chiba, sept.-29, Oct2. 1998, pp246-249

5. H. Wu, C. R. Elsass, A. Abare, M. Mack, S. Keller, P. M. Petroff, S. P. DenBaars, J. S. Speck, and S. J. Rosner Appl. Phys. Lett 72, pp.692-694,(1997).

6. T. Sugahara, M. Hao, T. Wang, D. Nakagawa, Y. Naoi, K. Nishino and S. Sakai Jpn.J.Appl. Phys., 37, pp.1195, (1998).

7. P. Li, S. J.Chua, G. Li, W.Wang, X.C. Wang, and Y. P. Guo, The Third International Conference on Nitride Semiconductor, Montpellier, France, July 4-july 9, 1999.

8. S.J. Chua, and G . Li to be published.

9.Yong-Hoon Cho, B.D. Little, G. H. Gainer, J. J. Song, S. Keller, U. K. Mishra, and S. P. Denbaars, Section G, GaN and related Materials, MRS Fall meeting 1998.

10. J. E. Northup, L. T. Romano and J. Neugebauer, Appl.Phys. Lett.,74,2319 (1999). 